\title{
A SUMMABILITY FACTOR THEOREM FOR ABSOLUTE SUMMABILITY INVOLVING ALMOST INCREASING SEQUENCES
}

\section{B. E. RHOADES and EKREM SAVAŞ}

Received 17 October 2003

We obtain sufficient conditions for the series $\sum a_{n} \lambda_{n}$ to be absolutely summable of order $k$ by a triangular matrix.

2000 Mathematics Subject Classification: 40G99, 40G05, 40D15.

A triangle is a lower triangular matrix with all principal diagonal entriesbeing nonzero. Given an almost increasing sequence $\left\{X_{n}\right\}$ and a sequence $\left\{\lambda_{n}\right\}$ satisfying certain conditions, we obtain sufficient conditions for the series $\sum a_{n} \lambda_{n}$ to be absolutely summable of order $k \geq 1$ by a triangle $T$. As a corollary we obtain the corresponding result when $T$ is a weighted mean matrix.

Theorem 1 of this paper is an example of a summability factor theorem. There is a large literature dealing with summability factor theorems. For example, MathSciNet lists over 500 papers dealing with this topic. For some other papers treating absolute summability factor theorems (of order $k \geq 1$ ) the reader may wish to consult [3, 4, 5].

Let $T$ be a lower triangular matrix,and $\left\{s_{n}\right\}$ a sequence. Then

$$
T_{n}:=\sum_{v=0}^{n} t_{n v} s_{v}
$$

A series $\sum a_{n}$, with partial sums $s_{n}$, is said to be summable $|T|_{k}, k \geq 1$ if

$$
\sum_{n=1}^{\infty} n^{k-1}\left|T_{n}-T_{n-1}\right|^{k}<\infty
$$

We may associate with $T$ two lower triangular matrices $\bar{T}$ and $\hat{T}$ as follows:

$$
\begin{aligned}
& \bar{t}_{n v}=\sum_{r=v}^{n} t_{n r} \quad n, v=0,1,2, \ldots, \\
& \hat{t}_{n v}=\bar{t}_{n v}-\bar{t}_{n-1, v} \quad n=1,2,3, \ldots
\end{aligned}
$$

We may write

$$
T_{n}=\sum_{v=0}^{n} t_{n v} \sum_{i=0}^{v} a_{i} \lambda_{i}=\sum_{i=0}^{n} a_{i} \lambda_{i} \sum_{v=i}^{n} t_{n v}=\sum_{i=0}^{n} \bar{t}_{n i} a_{i} \lambda_{i} .
$$


Thus

$$
\begin{aligned}
T_{n}-T_{n-1} & =\sum_{i=0}^{n} \bar{t}_{n i} a_{i} \lambda_{i}-\sum_{i=0}^{n-1} \bar{t}_{n-1, i} a_{i} \lambda_{i} \\
& =\sum_{i=0}^{n} \bar{t}_{n i} a_{i} \lambda_{i}-\sum_{i=0}^{n} \bar{t}_{n-1, i} a_{i} \lambda_{i} \\
& =\sum_{i=0}^{n}\left(\bar{t}_{n i}-\bar{t}_{n-1, i}\right) a_{i} \lambda_{i} \\
& =\sum_{i=0}^{n} \hat{t}_{n i} a_{i} \lambda_{i}=\sum_{i=0}^{n} \hat{t}_{n i} \lambda_{i}\left(s_{i}-s_{i-1}\right) \\
& =\sum_{i=0}^{n-1} \hat{t}_{n i} \lambda_{i} s_{i}-\sum_{i=0}^{n} \hat{t}_{n i} \lambda_{i} s_{i-1} \\
& =\sum_{i=0}^{n-1} \hat{t}_{n i} \lambda_{i} s_{i}+\hat{t}_{n n} \lambda_{n} s_{n}-\sum_{i=0}^{n} \hat{t}_{n i} \lambda_{i} s_{i-1} \\
& =\sum_{i=0}^{n-1} \hat{t}_{n i} \lambda_{i} s_{i}+t_{n n} \lambda_{n} s_{n}-\sum_{i=0}^{n-1} \hat{t}_{n, i+1} \lambda_{i+1} s_{i} \\
& =\sum_{i=0}^{n}\left(\hat{t}_{n i} \lambda_{i}-\hat{t}_{n, i+1} \lambda_{i+1}\right) s_{i}+t_{n n} \lambda_{n} s_{n} .
\end{aligned}
$$

We may write

$$
\begin{aligned}
\left(\hat{t}_{n i} \lambda_{i}-\hat{t}_{n, i+1} \lambda_{i+1}\right) & =\hat{t}_{n i} \lambda_{i}-\hat{t}_{n, i+1} \lambda_{i+1}-t_{n, i+1} \lambda_{i}+t_{n, i+1} \lambda_{i} \\
& =\left(\hat{t}_{n i}-\hat{t}_{n, i+1}\right) \lambda_{i}+t_{n, i+1}\left(\lambda_{i}-\lambda_{i+1}\right) \\
& =\lambda_{i} \Delta_{i} \hat{t}_{n i}+\hat{t}_{n, i+1} \Delta \lambda_{i} .
\end{aligned}
$$

Therefore

$$
\begin{aligned}
T_{n}-T_{n-1} & =\sum_{i=0}^{n-1} \Delta_{i} \hat{t}_{n i} \lambda_{i} s_{i}+\sum_{i=0}^{n-1} \hat{t}_{n, i+1} \Delta \lambda_{i} s_{i}+t_{n n} \lambda_{n} s_{n} \\
& =T_{n 1}+T_{n 2}+T_{n 3} .
\end{aligned}
$$

A positive sequence $\left\{b_{n}\right\}$ is said to be almost increasing if there exists a positive increasing sequence $\left\{c_{n}\right\}$ and two positive constants $A$ and $B$ such that $A c_{n} \leq b_{n} \leq B c_{n}$ for each $n$.

THeOREM 1. Let $\left\{X_{n}\right\}$ be an almost increasing sequence and let $\left\{\beta_{n}\right\}$ and $\left\{\lambda_{n}\right\}$ be sequences such that

(i) $\left|\Delta \lambda_{n}\right| \leq \beta_{n}$,

(ii) $\lim \beta_{n}=0$,

(iii) $\sum_{n=1}^{\infty} n\left|\Delta \beta_{n}\right| X_{n}<\infty$,

(iv) $\left|\lambda_{n}\right| X_{n}=O(1)$

are satisfied. 
Let $T$ be a triangle satisfying the following:

(v) $n t_{n n}=O(1)$,

(vi) $t_{n-1, v} \geq t_{n v}$ for $n \geq v+1$,

(vii) $\bar{t}_{n 0}=1$ for all $n$,

(viii) if $\sum_{n=1}^{m}(1 / n)\left|s_{n}\right|^{k}=O\left(X_{m}\right)$, then the series $\sum a_{n} \lambda_{n}$ is summable $|T|_{k}, k \geq 1$.

Proof. To prove the theorem it will be sufficient to show that

$$
\sum_{n=1}^{\infty} n^{k-1}\left|T_{n r}\right|^{k}<\infty \text { for } r=1,2,3 .
$$

From [2, page 86] it follows that (vi) and (vii) imply that

$$
\sum_{i=0}^{n-1}\left|\Delta_{i} \hat{t}_{n i}\right|=O\left(t_{n n}\right) .
$$

From (iv) and the fact that $\left\{X_{n}\right\}$ is almost increasing, it follows that $\lambda_{n}=O(1)$. Using Hölder's inequality, (9), and (v),

$$
\begin{aligned}
\sum_{n=1}^{m+1} n^{k-1}\left|T_{n 1}\right|^{k} & \leq \sum_{n=1}^{m+1} n^{k-1}\left(\sum_{i=0}^{n-1}\left|\Delta_{i} \hat{t}_{n i}\right|\left|\lambda_{i}\right|\left|s_{i}\right|\right)^{k} \\
& \leq \sum_{n=1}^{m+1} n^{k-1}\left(\sum_{i=0}^{n-1}\left|\Delta_{i} \hat{t}_{n i}\right|\left|\lambda_{i}\right|^{k}\left|s_{i}\right|^{k}\right)\left(\sum_{i=0}^{n-1}\left|\Delta_{i} \hat{t}_{n i}\right|\right)^{k-1} \\
& =O(1) \sum_{n=1}^{m+1}\left(n t_{n n}\right)^{k-1} \sum_{i=0}^{n-1}\left|\Delta_{i} \hat{t}_{n i}\right|\left|\lambda_{i}\right|^{k}\left|s_{i}\right|^{k} \\
& =O(1) \sum_{n=1}^{m+1}\left(n t_{n n}\right)^{k-1} \sum_{i=0}^{n-1}\left|\Delta_{i} \hat{t}_{n i}\right|\left|\lambda_{i}\right|\left|\lambda_{i}\right|^{k-1}\left|s_{i}\right|^{k} \\
& =O(1) \sum_{i=0}^{m}\left|\lambda_{i}\right|\left|s_{i}\right|^{k} \sum_{n=i+1}^{m+1}\left(n t_{n n}\right)^{k-1}\left|\Delta_{i} \hat{t}_{n i}\right| \\
& =O(1) \sum_{i=0}^{m}\left|\lambda_{i}\right|\left|s_{i}\right|^{k} t_{i i} \sum_{i=0}^{m}\left|\lambda_{i}\right|\left(\sum_{r=0}^{i}\left|s_{r}\right|^{k} t_{r r}-\sum_{r=0}^{i-1}\left|s_{r}\right|^{k} t_{r r}\right) \\
& \left.=O(1) \sum_{i=0}^{m-1}\left|\lambda_{i}\right| \sum_{r=0}^{i}\left|s_{r}\right|^{k} t_{r r}-\sum_{j=0}^{m}\left|\lambda_{j+1}^{j}\right| \sum_{r=0}^{j}\left|s_{r}\right|^{k} t_{r r}\right] \\
& =O(1)\left[\sum_{r=0}^{m-1} \Delta\left|\lambda_{i}\right| \sum_{r=0}^{i}\left|s_{r}\right|^{k} t_{r r}+\left|\lambda_{m}\right| \sum_{r=0}^{m}\left|s_{r}\right|^{k} t_{r r}\right] .
\end{aligned}
$$

If we define

$$
X_{i}=\sum_{r=0}^{i}\left|s_{r}\right|^{k} t_{r r}
$$


then $\left\{X_{i}\right\}$ is an almost increasing sequence. Using (iv) and (i),

$$
\sum_{n=1}^{m+1} n^{k-1}\left|T_{n 1}\right|^{k}=O(1) \sum_{i=0}^{m-1} \beta_{i} X_{i}+O(1)=O(1),
$$

using the result of [1, Lemma 3].

Using (i), Hölder's inequality, (ii), (v), (vi), and (vii),

$$
\begin{aligned}
& \sum_{n=1}^{m+1} n^{k-1}\left|T_{n 2}\right|^{k} \leq \sum_{n=1}^{m+1} n^{k-1}\left|\sum_{i=0}^{n-1} \hat{t}_{n, i+1} s_{i} \Delta \lambda_{i}\right|^{k} \\
& \leq \sum_{n=1}^{m+1} n^{k-1}\left(\sum_{i=0}^{n-1}\left|\hat{t}_{n, i+1}\right|\left|\Delta \lambda_{i}\right|\left|s_{i}\right|\right)^{k} \\
& \leq \sum_{n=1}^{m+1} n^{k-1}\left(\sum_{i=0}^{n-1}\left|\hat{t}_{n, i+1}\right|\left|s_{i}\right| \beta_{i}\right)^{k} \\
& \leq \sum_{n=1}^{m+1} n^{k-1} \sum_{i=0}^{n-1}\left|\hat{t}_{n, i+1}\right|\left|s_{i}\right|^{k} \beta_{i}\left(\sum_{i=0}^{n-1}\left|\hat{t}_{n, i+1}\right| \beta_{i}\right)^{k-1} \\
& =O(1) \sum_{n=1}^{m+1}\left(n t_{n n}\right)^{k-1} \sum_{i=0}^{n-1}\left|\hat{t}_{n, i+1}\right|\left|s_{i}\right|^{k} \beta_{i} \\
& =O(1) \sum_{i=1}^{m} \beta_{i}\left|s_{i}\right|^{k} \sum_{n=i+1}^{m+1}\left|\hat{t}_{n, i+1}\right| \\
& =O(1) \sum_{i=1}^{m} \beta_{i}\left|s_{i}\right|^{k}=O(1) \sum_{i=1}^{m} i \beta_{i} \frac{1}{i}\left|s_{i}\right|^{k} \\
& =O(1) \sum_{i=1}^{m} \beta_{i}\left[\sum_{r=1}^{i} \frac{\left|s_{r}\right|^{k}}{r}-\sum_{r=1}^{i-1} \frac{\left|s_{r}\right|^{k}}{r}\right] \\
& =O(1)\left[\sum_{i=1}^{m} i \beta_{i} \sum_{r=1}^{i} \frac{\left|s_{r}\right|^{k}}{r}-\sum_{j=1}^{m-1}(j+1) \beta_{j+1} \sum_{r=1}^{j} \frac{\left|s_{r}\right|^{k}}{r}\right] \\
& =O(1) \sum_{i=1}^{m-1} \Delta\left(i \beta_{i}\right) \sum_{r=1}^{i} \frac{1}{r}\left|s_{r}\right|^{k}+O(1) m \beta_{m} \sum_{i=0}^{m} \frac{1}{i}\left|s_{i}\right|^{k} \\
& =O(1) \sum_{i=1}^{m-1}\left|\Delta\left(i \beta_{i}\right)\right| X_{i}+O(1) m \beta_{m} X_{m} \\
& =O(1) \sum_{i=0}^{m-1} i\left|\Delta\left(\beta_{i}\right)\right| X_{i}+O(1) \sum_{i=0}^{m-1} \beta_{i} X_{i}+O(1) m \beta_{m} X_{m}=O(1) \text {, }
\end{aligned}
$$

again using [1, Lemma 3]. 
Using (v),

$$
\begin{aligned}
\sum_{n=1}^{m+1} n^{k-1}\left|T_{n 3}\right|^{k} & \leq \sum_{n=1}^{m+1} n^{k-1}\left|t_{n n} \lambda_{n} s_{n}\right|^{k} \\
& =O(1) \sum_{n=1}^{m}\left(n t_{n n}\right)^{k-1} t_{n n}\left|\lambda_{n}\right|^{k}\left|s_{n}\right|^{k} \\
& =O(1) \sum_{n=1}^{m} t_{n n}\left|\lambda_{n}\right|^{k-1}\left|\lambda_{n}\right|\left|s_{n}\right|^{k}=O(1),
\end{aligned}
$$

as in the proof of $T_{n 1}$.

A weighted mean matrix, denoted by $\left(\bar{N}, p_{n}\right)$, is a lower triangular matrix with nonzero entries $p_{k} / P_{n}$, where $\left\{p_{n}\right\}$ is a nonnegative sequence with $p_{0}>0$ and $P_{n}:=$ $\sum_{i=0}^{n} p_{i} \rightarrow \infty$ as $n \rightarrow \infty$.

COROLlARY 2. Let $\left\{X_{n}\right\}$ be an almost increasing sequence and let condition (viii) of Theorem 1 be satisfied. If $\left\{\beta_{n}\right\}$ and $\left\{\lambda_{n}\right\}$ satisfy conditions (i)-(iv) of Theorem 1 and if $\left\{p_{n}\right\}$ is a sequence such that

(i) $n p_{n} / P_{n}=O(1)$,

then the series $\sum a_{n} \lambda_{n}$ is summable $\left|\bar{N}, p_{n}\right|_{k}, k \geq 1$.

Proof. With $T=\left(\bar{N}, p_{n}\right)$, condition (v) of Theorem 1 reduces to condition (i). Conditions (vi) and (vii) of Theorem 1 are automatically satisfied.

It should be noted that, in [1], an incorrect definition of absolute summability was used (see, e.g., [2]). Corollary 2 gives the correct version of Bor's theorem.

ACKNOWLEDGMENT. This research was completed while the second author was a Fulbright scholar at Indiana University during the fall semester of 2003.

\section{REFERENCES}

[1] H. Bor, An application of almost increasing sequences, Int. J. Math. Math. Sci. 23 (2000), no. $12,859-863$.

[2] B. E. Rhoades, Inclusion theorems for absolute matrix summability methods, J. Math. Anal. Appl. 238 (1999), no. 1, 82-90.

[3] B. E. Rhoades and E. Savaş, Some necessary conditions for absolute matrix summability factors, Indian J. Pure Appl. Math. 33 (2002), no. 7, 1003-1009.

[4] _ Some summability factor theorems for absolute summability, Analysis (Munich) 22 (2002), no. 2, 163-174.

[5] _ A summability type factor theorem, Tamkang J. Math. 34 (2003), no. 4, 395-401.

B. E. Rhoades: Department of Mathematics, Indiana University, Bloomington, IN 47405-7106, USA

E-mail address: rhoades@indiana.edu

Ekrem Savaş: Department of Mathematics, Yüzüncü Yil University, 65080 Van, Turkey

E-mail address: ekremsavas@yahoo.com 


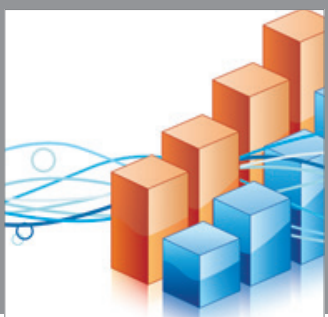

Advances in

Operations Research

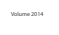

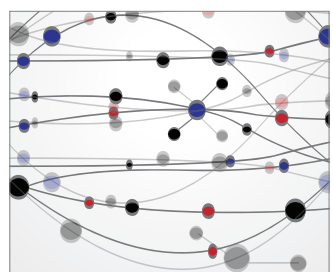

\section{The Scientific} World Journal
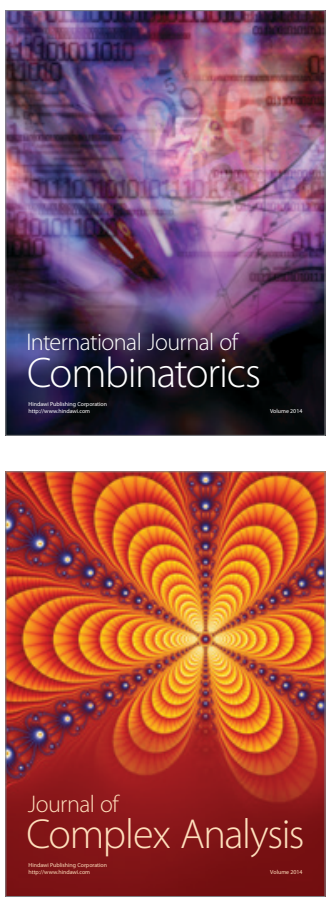

International Journal of

Mathematics and

Mathematical

Sciences
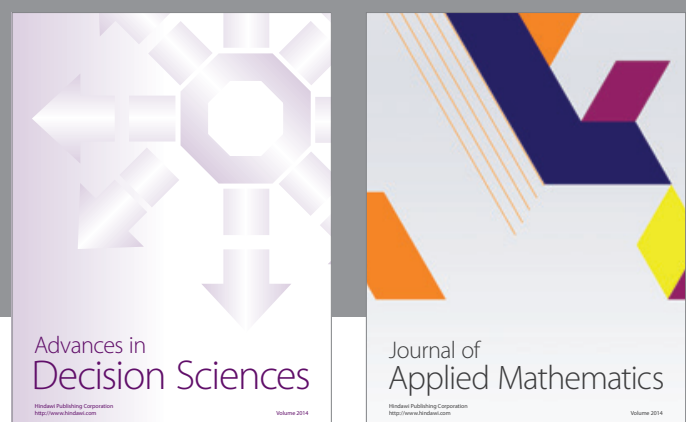

Journal of

Applied Mathematics
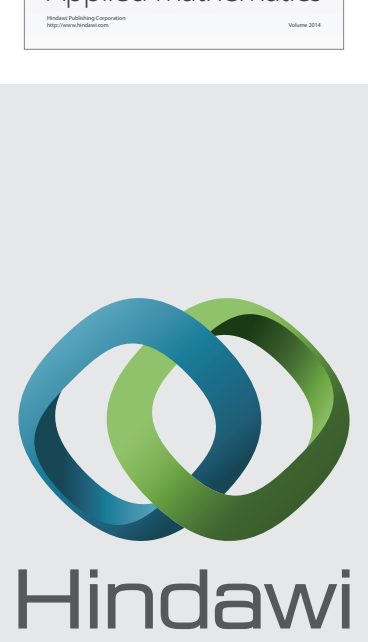

Submit your manuscripts at http://www.hindawi.com
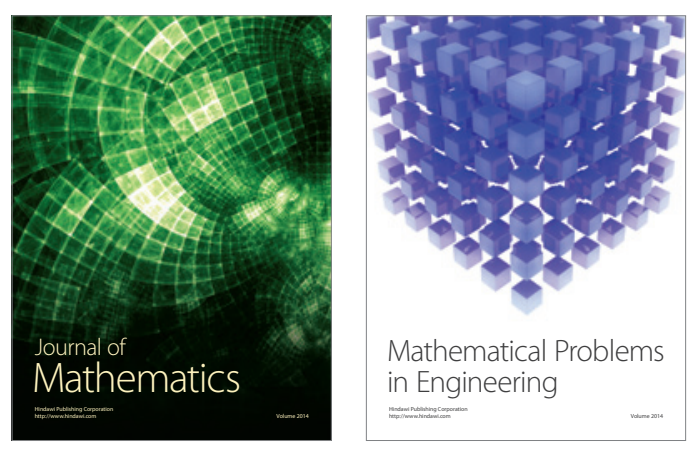

Mathematical Problems in Engineering
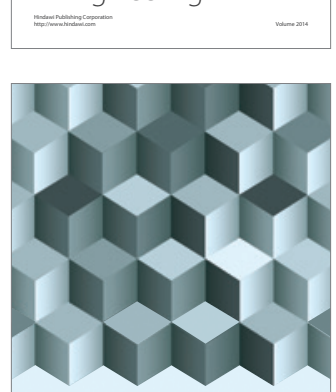

Journal of

Function Spaces
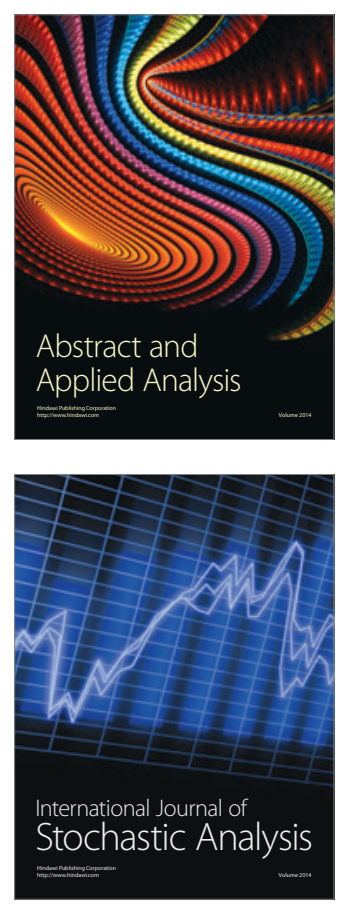

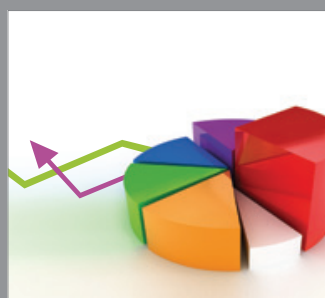

ournal of

Probability and Statistics

Promensencen
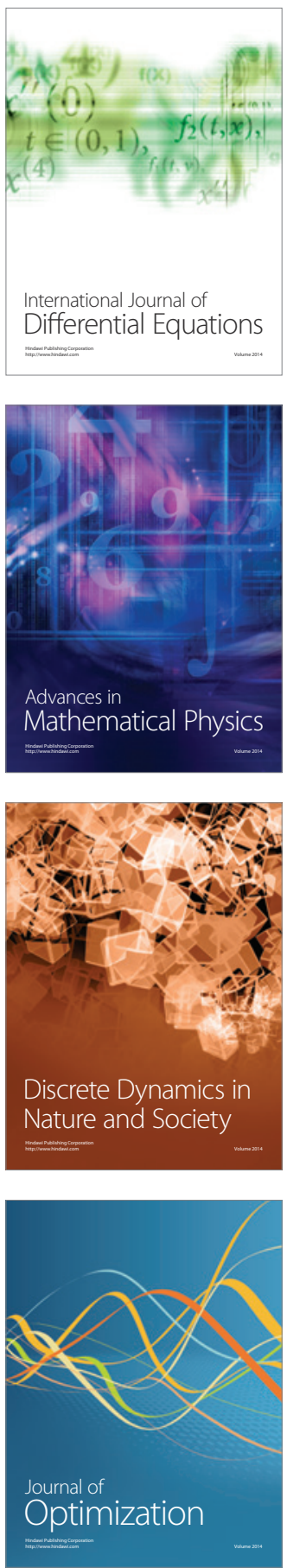